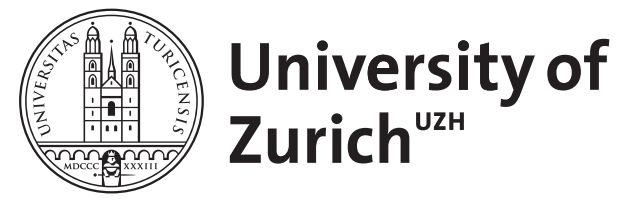

Zurich Open Repository and Archive

University of Zurich

University Library

Strickhofstrasse 39

CH-8057 Zurich

www.zora.uzh.ch

Year: 2012

\title{
Sozialunternehmen und die Reintegration der losgelösten Wirtschaft in ihren sozio-normativen Kontext
}

\author{
Gonin, Michael
}

Posted at the Zurich Open Repository and Archive, University of Zurich

ZORA URL: https://doi.org/10.5167/uzh-63232

Book Section

Published Version

Originally published at:

Gonin, Michael (2012). Sozialunternehmen und die Reintegration der losgelösten Wirtschaft in ihren sozio-normativen Kontext. In: Pfleiderer, Georg; Seele, Peter. Wirtschaftsethik kontrovers. Positionen aus Theorie und Praxis. Zurich: Nomos Verlag, 103-118. 


\section{Sozialunternehmen und die Reintegration der losgelösten Wirtschaft in ihren sozio-normativen Kontext}

\section{Michaël Gonin}

\section{Einleitung}

Oft wird auf Adam Smith verwiesen, um die heutige neoliberale Marktwirtschaft zu rechtfertigen. Jedoch wird gleichzeitig übersehen, dass die politische und gesellschaftliche Ordnung in Smiths Zeit nicht derjenigen der heutigen globalen und pluralistischen Welt entspricht. Vor allem war das Unternebmen nicht als Person anerkannt, wie es heute bei Aktiengesellschaften der Fall ist. Es blieb das Projekt einer Person - das heißt, es konnte nicht unabhängig von einem Unternehmer existieren. Diese Person investierte Zeit, Geld, und Energie darin, und identifizierte sich mit ihrer Arbeit. Ferner war sie Mitglied einer lokalen Gesellschaft und teilte dadurch gemeinsame Werte und Sitten mit den Mitbürgern.

In einem solchen Kontext können sich wirtschaftliche Tätigkeiten nicht von der Kontrolle der Gesellschaft loslösen. Heutzutage wird aber (größeren) Unternehmen oft vorgeworfen, sie seien dem normativen Rahmen der Gesellschaft nicht mehr treu. Gleichzeitig erhalten Sozialunternebmen größere Beachtung und Legitimität wegen ihrer Fähigkeit, in wirtschaftlich effizienter Weise wichtige gesellschaftliche Ziele zu verfolgen.

Dieses Kapitel geht der Frage nach, inwiefern das Konzept des Sozialunternehmens die heutige Version des ursprünglichen eingebetteten Unternehmens darstellt - und dadurch zur Überwindung der immer stärker kritisierten Trennung der wirtschaftlichen und gesellschaftlichen Sphären beitragen kann. Zuerst werden Kontext und Charakteristiken des (idealtypischen) eingebetteten Unternehmens vorgestellt und der Loslösungsprozess besprochen. Der zweite Teil geht der Frage nach, inwiefern die schweizerischen Sozialunternehmen zur Reintegration der Wirtschaft in die Gesellschaft beitragen können. 


\section{Die eingebettete Wirtschaft}

Adam Smith hat die heutige Gesellschaft und insbesondere die Wirtschaft stark beeinflusst. Oft wird aber nur eine seiner Behauptungen zitiert: «Nicht vom Wohlwollen des Metzgers, Brauers und Bäckers erwarten wir das, was wir zum Essen brauchen, sondern davon, daß sie ihre eigenen Interessen wahrnehmen.»1

Dieses Zitat ist aber im Kontext der damals stärker eingebetteten Gesellschaft zu verstehen. Die Wirtschaft war zu Smiths Zeit ein integraler Bestandteil der Gesellschaft, und dadurch dem gesellschaftlichen normativen Rahmen unterstellt. Etwas pointiert gesagt wurde jedes Handeln, sei es wirtschaftlicher, sozialer, kultureller, religiöser, politischer, oder sogar privater Natur, überprüft und gegebenenfalls diskutiert, kommentiert und beurteilt. Fehlverhalten in einer Lebenssphäre (zum Beispiel der wirtschaftlichen) führte zur Benachteiligung der Person in (allen) anderen Lebenssphären. Wer in ein unfaires Geschäft involviert war, musste mit Bemerkungen während der Chor- oder Feuerwehrübung und einer expliziten oder stillschweigenden Rüge während der nächsten Predigt rechnen...

In einem solchen Kontext stellt die moralische Billigung von Handlungen durch die breitere Gemeinschaft eine Bedingung für soziale Anerkennung und damit für die Eingliederung in der Gesellschaft dar. Eigeninteresse kann nicht so egozentrisch und gierig verstanden werden, wie es heutzutage in der Wirtschaftstheorie interpretiert wird. Nicht dass die Akteure in einer normativ starken Gesellschaft keine eigenen Interessen haben. Ihr Eigeninteresse ist aber stark durch soziale und moralische Normen geprägt und abgemildert. Metzger, Brauer und Bäcker bleiben in erster Linie Mitbürger, die aufgrund ihrer menschlichen Natur «an dem Schicksal anderer Anteil» ${ }^{2}$ nehmen und von der (moralischen) Anerkennung ihrer Gemeinschaft abhängig sind.

Dieser starke Rahmen, zusammen mit protektionistischen Maßnahmen und begrenzten Transportmöglichkeiten, begünstigt lokal verankerte Familienbetriebe. Größere Firmen stellen Ausnahmen dar und bleiben auf

1 Smith, Adam, Der Wohlstand der Nationen. Eine Untersuchung seiner Natur und seiner Ursachen (Übers: H.C. Recktenwald), München, Beck Verlag, 1974 (1789), Kapitel I.ii.

2 Smith, Adam, Theorie der ethischen Gefühle (Übers: W. Eckstein), Hamburg, Meiner, 2004 (1790), allererster Absatz. 
jeden Fall dem lokalen Leben untertan. Trotz internationalem Geschäft blieben zum Beispiel die einzelnen Familienmitglieder des Medici-Imperiums in Florenz verankert. Sie hatten einen gewissen Einfluss auf das politische Leben der Stadt und der Gegend, waren aber von den Sitten und Normen der Stadt stark geprägt. ${ }^{3}$

\section{Die implizite Unterordnung der Wirtschaft unter gesellschaftliche Normen}

In einem starken Kontext kann das Unternehmen nichts anderes sein als eine konkrete Handlung einzelner Unternehmer, die sich mit ihrem Projekt identifizieren und selbst Geld und Zeit darin investieren. Diese Unternehmer sind gleichzeitig Eigentümer ihrer Geschäfte (so fern man ein Projekt 'besitzen' kann) und Mitbürger einer Gesellschaft. Diese Einbettung kann durch folgende ideal-typische Gleichung zusammengefasst werden:

$$
\text { Unternehmen } \Leftrightarrow \text { Unternehmer } \Leftrightarrow \text { Eigentümer } \Leftrightarrow \text { Bürger }
$$

Eine solche Einbettung des wirtschaftlichen Handelns kann in den meisten Grundtexten der westlichen Geschichte gefunden werden. Neben der oben erwähnten Weltanschauung von Adam Smith ist zum Beispiel diejenige von Aristoteles bemerkenswert: Aristoteles unterscheidet zwischen den ökonomischen Aktivitäten eines Haushalts innerhalb der lokalen Gemeinschaft (oikonomia) und den Geschäften der cbrematistike. Während erstere zur Selbsterhaltung des (breiteren) Haushalts dienen und durch die Sitten der lokalen Gemeinschaft geregelt sind, sind letztere zu stark giergetrieben, um moralisch gebilligt zu werden. Ähnliche Ideen können ferner in der christlichen Tradition gefunden werden. Vom Ruhetaggebot bis zur Fürsorge der Schwächsten und von Jesu Gebot, seinen zweiten Mantel zu schenken, ${ }^{4}$ falls jemand keinen hat bis hin zur Idee, dass jede Familie regelmäßig wieder ihre 'Produktionsmittel' (damals das Familienlandgut) zurückkriegt - die Grundtexte des Christentums betrachten schon damals Wirtschaft als eine stark regulierte Dienerin des persönlichen und gemeinschaftlichen Wohls.

3 Siehe Cleugh, James, The Medici. A Tale of Fifteen Generations, New York, Doubleday \& Company, 1975.

4 Es muss festgestellt werden, dass Jesus nichts zum Falle sagt, jemand hätte hundert Mantel und alle andere doch mindestens einen. 
Klar sind in stark geregelten Kulturen auch gierige und sonst moralisch fragwürdige Personen zu finden. Sie werden aber als krankhafte Charaktere von den Mitbürgern moralisch verurteilt. Die allgegenwärtigen gesellschaftlichen Normen, zusammen mit der gegenseitigen Überwachung der Bürger, prägen alle Lebenssphären.

Im nächsten Teil wird aber gezeigt, dass im 20. Jahrhundert die Gleichung aus verschiedenen Gründen auseinanderbrach. Diese Änderung fuhr zu einer Loslösung des Unternehmens vom impliziten Einfluss der gesellschaftlichen Normen auf das Wirtschaftsleben.

\section{Die Loslösung der Wirtschaft von ihrem sozialen Rahmen ${ }^{5}$}

\section{Das Aufkommen der juristischen Person}

Mit der Zeit hat sich das Unternehmensverständnis als das konkrete Handeln eines Bürgers geändert, um eher eine selbständige Organisation darzustellen. Diese Verselbstständigung des Unternehmens fing mit der Entwicklung der Aktiengesellschaft als Organisationsform an. So wurde im 17. Jahrhundert die East India Company zur ersten unbefristeten, nicht projektgebundenen Aktiengesellschaft umgewandelt.

$\mathrm{Ab}$ dem 19. Jahrhundert bezeichnet der Begriff «juristische Person» immer öfter die Organisation - trotz mangelnder rechtlicher Grundlage. ${ }^{6}$ Dadurch werden Aktiengesellschaften zu Akteuren mit eigenständigem Willen und eingeständigen Handlungsmöglichkeiten. Die erste Gleichung, Unternebmen = Unternehmer, wird aufgebrochen. Die juristische Person bildet heutzutage eine spezifische Gruppe in der Gesellschaft, die nicht derjenigen der natürlichen Personen entspricht, die jedoch gleich wie natürliche Personen wirtschaftlich tätig sein kann.

5 Dieser Text bezieht sich zum Teil wörtlich auf einem früheren Beitrag des Autors: Gonin, Michaël, Die Trennung des Unternehmens vom Bürger - eine historischkritische Perspektive, in: Patry, Eric/Ulrich, Peter, Hg., Marktwirtschaft als Teil einer bürgergesellschaftlichen Gesamtordnung (Berichte des Instituts für Wirtschaftsethik Nr. 107), St. Gallen, Institut für Wirtschaftsethik der Universität St. Gallen, 2006, 41-56.

6 Der Begriff wurde zum ersten Mal in einem Gerichtsurteil genutzt. Vgl. Nace, Ted, Gangs of America. The Rise of Corporate Power and the Disabling of Democracy, San Francisco, Berrett-Koehler Publishers, 2003, 102-109. 
Da sie aber keine natürlichen Personen sind, fehlt den juristischen Personen die oben erwähnten natürlichen Neigungen, im wirtschaftlichen Handeln eine Bürgertugend zu integrieren und die (moralische) Akzeptanz der breiteren Gesellschaft zu suchen. Die Ziele und Werte juristischer Personen werden nicht von einer Mitbürgerschaft in einer Gesellschaft geprägt, sondern künstlich durch ihre eigene interne (Wirtschafts-)logik definiert. Statuten, Leitbilder und Verhaltenskodizes diktieren, wofür die Organisation steht.

Sicher werden diese Dokumente innerhalb der Organisation von Menschen erstellt. In den nächsten Abschnitten soll aber gezeigt werden, dass diese Menschen spezifische, vom Bürgerethos losgelöste Rollen übernehmen.

\section{Die Abtrennung des Unternehmers von den gesellschaftlichen Normen}

Während des 20. Jahrhunderts ersetzt der Manager zunehmend den Unternehmer in etablierten Unternehmen. Management wird zu eigenständigem Beruf, der von spezifisch ausgebildeten Personen betrieben wird. Diese Akteure übernehmen eine klar definierte und begrenzte Rolle. Laut der dominierenden Principal-Agent Theory ${ }^{7}$ sind Manager Beauftragte, die durch angepasste Anreizsysteme dazu geführt werden sollen, die Ziele der Eigentümer zu erreichen. Von Managern wird nicht erwartet, dass sie als autonome Personen handeln. Als Agenten vertreten sie das Unternehmen und funktionieren als «Wirtschaftsautokraten» ${ }^{8}$, die die Profite der Aktionäre maximieren. Ihre persönlichen Werte sollen ihre wirtschaftliche Funktion nicht beeinflussen. ${ }^{9}$ Konsequenterweise können Manager als

7 Vgl. Rees, Ray, The theory of principal and agent: Part 1 \& 2, Bulletin of Economic Research, 37(1), 1985, 3-26 \& 37(2), 75-95. Friedman hat diese Idee durch seine pointierte Formulierung als Titel eines Artikels deutlich gemacht: Friedman, Milton, The social responsibility of business is to increase its profits, New York Times Magazine, 1970 (September 13 ${ }^{\text {th }}$ ), 122-126.

8 Vgl. Berle, Adolf A./Means, Gardiner C., The Modern Corporation \& Private Property, London, Transaction Publishers, 2005 [1932], 116.

9 Vgl. Mintzberg, Henry, Manager statt MBAs: Eine kritische Analyse, Frankfurt am Main, Campus Verlag, 2005 sowie den Fallbeispiel in Pruzan, Peter, The question of organizational consciousness: Can organizations have values, virtues and visions?, Journal of Business Ethics, 29(3), 2001, 271-284.. 
«ethisch stumme» Akteure betrachtet werden, ${ }^{10}$ die während der Arbeit ihre zivilgesellschaftlichen Verantwortungen nicht beachten sollen.

In der Principal-Agent Theory wird die Autokratisierung des Managements und die daraus folgende ethische Verstummung der Akteure dadurch gerechtfertigt, dass die Eigentümer, als Principal, die Grundwerte definieren und durch geeignete Mittel das Verhalten der Organisationsmitglieder lenken sollen. Im nächsten Teil werden aber mehrere Faktoren diskutiert, die die Eigentümer von ihren Bürgerverantwortungen ablenken.

\section{Die Abtrennung des Eigentümers von der gesellschaftlichen Normen}

Mehrere Gründe führen zu einem begrenzten Bürgerethos bei Eigentümern. Erstens ist es generell bekannt, dass je mehr Personen für etwas verantwortlich sind, desto weniger sich jede einzelne Person dafür verantwortlich fühlt. Die drastische Erhöhung der Anzahl Aktionäre hat also dazu geführt, dass das Verantwortungsgefühl sank. Wenn hunderttausende von Personen je einen Bruchteil ihres Kapitals anonym in einer Firma investieren, fehlt eine Identifikation der einzelnen Eigentümer mit dem Unternehmen. ${ }^{11}$

Das Verantwortungsgefühl wird ferner durch Kapitalverschachtelung den Kauf von Aktien durch juristische Personen - und die Entwicklung der institutionellen Investoren nochmals reduziert. Die Mehrheit der Aktien der größeren Unternehmen gehört nämlich heutzutage wirtschaftlichen Institutionen. Dadurch wird aber der bürgerliche Eigentümer nochmals vom Unternehmen entfernt. Die Eigentümer von Unternehmen sind keine Menschen mehr, sondern selbst juristische Personen. Als solche folgen sie ihren eigenen Zielen und Entscheidungsregeln. Diese Routinen werden

10 Bird, Frederick B./Waters, James A., The moral muteness of managers, California Management Review, 32(1), 1989, 73-88.

11 Betreffend dieses psychologische Phänomen, siehe Liden, Robert C./Wayne, Sandy J./Jaworski, Renata A./Bennett, Nathan, Social loafing: A field investigation, Journal of Management, 30(2), 2004, 285-304. Siehe auch die Unternehmensanalysen von Steinmann, Horst, Das Grossunternehmen im Interessenkonflikt. Ein wirtschaftswissenschaftlicher Diskussionsbeitrag zu Grundfragen einer Reform der Unternehmensordnung in hochentwickelten Industriegesellschaften, Stuttgart, C.E. Poeschel Verlag, 1969 sowie die juristische Diskussion in Pfister, Loïc, Les sociétés ouvertes au public et l'ordre constitutionnel, Genève, Schulthess, 2010. 
nicht durch das Bürgerethos (Unternehmen sind keine Bürger), sondern durch die Standard-Logik der ökonomischen Modelle definiert. ${ }^{12}$

Obwohl man argumentieren könnte, dass die institutionellen Anleger selbst Aktionäre haben, die schlussendlich natürliche Personen sind, vergrößert sich die Distanz zwischen den Menschen und dem konkreten Handeln der Firmen so sehr, dass das Bürgerethos verloren geht. Zum Beispiel sind sich die meisten Angestellten nicht bewusst, dass ihre Vorsorgestiftungen ihr Geld in die verschiedensten Firmen investieren. Durch Vorsorgepläne werden sie also indirekt zu Firmen-Eigentümer und sollten sich laut Principal-Agent Theorie um die Grundwerte der Firmen unter ihrem Besitz ${ }^{13}$ kümmern.

\section{Fazit: Das losgelöste und wertlose Unternehmen}

Die Verselbständigung des Unternehmens, die Herabsetzung der Unternehmensleitung zu einer engen, vordefinierten Management-Rolle und die Vermehrung und Distanzierung der Eigentümer vom täglichen Geschäft führen zur Abschaffung der Brücken zwischen dem Bürger und den verschiedenen wirtschaftlichen Akteuren. Die Vermittlung der gesellschaftlichen Werte vom Bürger zum Eigentümer-Unternehmer und davon zum Unternehmen ist damit durchbrochen. Das Eigeninteresse einer losgelösten juristischen Person, die ihrer Natur wegen keine moralische Billigung der breiteren Gesellschaft sucht, entspricht nicht demjenigen einer natürlichen Person. Ferner führt die Reduktion der Managementaufgabe auf eine rein wirtschaftliche Rolle zur moralischen Verstummung der Manager. Schlussendlich nehmen die unzähligen und fernen Aktieneigentümer ihre Verantwortung für die Werte 'ihrer' Firma nicht wahr.

Auf Smith zurückzukommen, um das losgelöste Wirtschaftssystem zu rechtfertigen ist also sicher interessant. Ein umfassenderes Verständnis des Kontexts, in welchem Smith schrieb, führt aber eher zu Mäßigung und Bürgertugend, anstatt zu bloßem 'Eigeninteresse' im wirtschaftlichen Handeln.

12 Vgl. z.B. Moldoveanu, Mihnea, Language, games and language games, Journal of SocioEconomics, 31(3), 2002, 233-251 sowie Ulrich, Peter, Integrative Wirtschaftsethik. Grundlagen einer lebensdienlicher Ökonomie, Bern, Haupt, 2001, 137-148.

13 Streng genommen kann eine Firma nicht 'besessen' werden, sondern nur ihre Aktien. Siehe Pfister, Sociétés et ordre constitutionnel, 96-100. 
Eine solche geistige Haltung ist heutzutage noch bei vielen Akteuren zu finden. Im nächsten Teil wird das Sozialunternehmertum als eine der aktuellen Verwirklichungen dieses Bürgerethos diskutiert.

\section{Sozialunternehmen und bürgerliches Denken}

\section{Das Sozialunternebmen als heutiger Prototyp eines bürgerlichen Wirtschaftsakteurs}

In den letzten Jahren wurde mehr und mehr über Sozialunternebmen und Sozialunternebmer berichtet. Damit ist eine Person oder Organisation gemeint, die als Kern ihres wirtschaftlichen Projekts explizit die Lösung eines gesellschaftlichen Problems setzt.

Wie traditionelle Firmen weisen Sozialunternehmen ein professionelles Management und das Bestreben nach Effizienz auf sowie die Notwendigkeit eines begrenzten Profits oder zumindest keines Verlusts. Sie sind auch keine öffentliche Institution, sondern entstehen und leben durch den Willen unternehmerischer Bürger. Sie können Geld vom Staat im Rahmen eines Dienstleistungsvertrags erhalten, zum Beispiel im Rahmen beruflicher Wiedereingliederungsprogramme. Solche vertraglichen Beziehungen sind aber grundsätzlich nicht von denjenigen zu unterscheiden, die andere Akteure wie IT-Dienstleistungs- oder Straßenbau-Firmen mit dem Staat unterhalten.

Sozialunternehmen und Sozialunternehmer unterscheiden sich aber von traditionellen Wirtschaftsakteuren durch mehrere Merkmale, die der oben beschriebene Bürger auch vorweist. Erstens verfolgen sie Ziele, die von der Gesellschaft geschätzt sind und der Gesellschaft direkt dienen. In dieser Perspektive setzen sie Eigeninteresse nicht mit Gier oder Profitmaximierung gleich. Eher geht es darum, eine finanzielle Abhängigkeit durch den kostendeckenden Verkauf von Gütern und Dienstleistungen zu vermeiden. ${ }^{14}$

Ferner unterstellen diese Wirtschaftsakteure ihre Projekte explizit den Normen der breiteren Gesellschaft. Sowohl das Ziel wie auch die Mittel werden den gesellschaftlichen Erwartungen angepasst. Für mehrere dieser

14 Siehe Yunus, Muhammad, Building Social Business: The New Kind of Capitalism That Serves Humanity's Most Pressing Needs, New York, PublicAffairs, 2010. 
Akteure ist auch Transparenz - das heißt, die Veröffentlichung der wichtigsten Zahlen, der Führungs- und Entscheidungsstruktur, sowie der finanziellen oder gesetzlichen Abhängigkeiten - Bestandteil ihrer Kultur. ${ }^{15}$

Schlussendlich verzichten gewisse Sozialunternehmen auf Wachstum und beharren auf einer lokalen Verankerung. Ihre Geschäftgröße soll die Pflege zwischenmenschlicher Beziehungen erlauben und lokale Partner werden oft bei Herstellung und Vertrieb vorgezogen. In dieser Hinsicht kommt Menschenwürde vor business.

\section{Geschichte und Zukunft der Sozialunternehmen in der Westschweiæ: Ein Überblick.}

Dass heutzutage viele Sozialunternehmen gegründet werden und dass sie mehr Aufmerksamkeit erhalten, ist durch die Kombination mehrerer Faktoren zu erklären. Vor allem ist aber zu beachten, dass der heutige Trend auf einer langen Tradition von Unternehmen mit einem Bürgerethos beruht. Sozialunternehmertum stellt keine neue Erfindung dar, sondern eher eine Wiederbelebung und Anpassung einer geistigen Haltung, die bis zum Anfang des 20. Jahrhunderts weit verbreitet war.

Im 19. Jahrhundert waren nämlich Aktiengesellschaften viel weniger präsent als im letzten Jahrhundert. Laut den Handelsregistern der sieben französischsprachigen Kantone ${ }^{16}$ wurden 1890-1899 mehr Genossenschaften gegründet als Aktiengesellschaften. ${ }^{17}$ Dieser Unterschied juristischer Natur spiegelt einen wichtigen Unterschied kultureller Natur wider. Genossenschaften werden nämlich nach dem demokratischen Motto 'Eine Stimme pro Person' und nicht nach Kapitalanteil wie in Aktiengesellschaften geführt. Ferner funktionieren sie nach dem Prinzip der «gemeinsamen Selbsthilfe» und dürfen den Eintritt neuer Mitglieder nicht prinzipiell verbieten. ${ }^{18}$

Viele dieser älteren Organisationen sind heutzutage noch $\mathrm{zu}$ finden. Neben Coop, Migros oder der Raiffeisenbank, die regelmäßig zitiert werden,

15 Vgl. die Definition des europäischen Forschungsnetwerks EMES unter www.emes.net.

16 Genf, Waadtland, Neuenburg, Jura sowie die drei zweisprachigen Kantonen Wallis, Freiburg und Bern.

17 Gonin, Michael/Gachet, Nicolas, A Two-Dimensional Mapping of Socio-Economic Organizations, presented at the 3rd EMES Conference, Roskilde, DK, 2011. 2000-2010 wurde nur noch eine Genossenschaft pro hundert Aktiengesellschaften gegründet.

18 Siehe das Schweizerische Obligationenrecht, unter anderem die Art. 828, 839, 885 
soll hier besonders Die Mobiliar erwähnt werden. 1826 gegründet, trat sie 2011 der Genfer Kammer der sozialen und solidarischen Wirtschaft ${ }^{19}$ bei und bekräftigte somit ihre Bürgeridentität neu.

Seit einigen Jahren können zusätzlich viele neue SozialunternehmenInitiativen beobachtet werden. Viele individuelle Personen haben aus verschiedensten Gründen die moralische Verankerung ihres Unternehmens der Sicherheit traditioneller Karrieren vorgezogen. ${ }^{20}$ Unternehmen im Bereich des Verkehrs, des lokalen und biologischen Konsums, der Wiederverwertung, der Finanzdienstleistungen, des Hotelgewerbes, der Versicherung und Vorsorge, aber auch Wiedereingliederungsfirmen aller Art, Restaurants und Partyservices, Konditoreien, Wohngenossenschaften in allen Wirtschaftsbereichen kann ein Zuwachs an Akteuren beobachtet werden.

Dieses Wachstum wird zusätzlich durch einen Kulturwechsel in vielen traditionellen gemeinnützigen Organisationen gestärkt. Diese weisen heutzutage ein stärkeres unternehmerisches Denken auf, zum Teil wegen dem staatlichen Druck, zum Teil wegen einer neuen Management-Kultur im Drittsektor. ${ }^{21}$ Anstatt nur auf Subventionen oder Spenden zu zählen, schaffen diese Organisationen Innovationen in den Bereichen des Produktoder Dienstleistungsangebots und der Geldquellen. Durch diese Managerialisierung 22 nähern sich also die Nonprofit-Organisationen dem unternehmerischen Geist der (Sozial-)unternehmen an und erhöhen die Sichtbarkeit dieser wirtschaftlichen Alternative in der Öffentlichkeit.

Die Sichtbarkeit und Legitimität dieses alternativen Geschäftsmodells wurde 2006 durch das Verleihen des Nobelpreises an Mubummad Yunus nochmals erhöht. ${ }^{23}$ Yunus bekam die Anerkennung für seinen Beitrag zur Armutsverminderung durch Mikrofinanz und für die Anpassung

19 Siehe unten.

20 Siehe zum Beispiel Bornstein, David, Die Welt verändern. Social Entrepreneurs und die Kraft neuer Ideen, Stuttgart, Klett-Cotta, 2009.

21 Siehe Schwartz, Peter/Purtschert, Robert/Giroud, Charles/Schauer, Reinbert, Das Freiburger Management-Modell für Nonprofit-Organisationen, Bern, Haupt, 2002 sowie die Analyse in Dey, Pascal, Below or Beyond 'Social Entrepreneurship'? Hegemonic Discourse and Transgressive Possibilities in Swiss Nonprofit Organisations. Presented at the $7^{\text {th }}$ International Critical Management Studies Conference, Naples, 2011.

22 Ob diese Änderung gut oder schlecht ist, ist nicht hier zu beurteilen.

23 Vgl. Yunus, Social Business. 
traditioneller Produkte und Dienstleistungen an die Bedürfnisse und finanziellen Möglichkeiten der Ärmsten. Obwohl es sich interessanterweise nicht um den Wirtschaftsnobelpreis handelte, unterstreicht diese Auszeichnung das Sozialunternehmertum als ernsthaften 'dritten Weg' zwischen dem moralisch stummen freien Markt einerseits und dem gescheiterten Kommunismus andererseits. ${ }^{24}$

\section{Die mangelnde Institutionalisierung der Sozialunternehmen in der Schweiz.}

Auf institutioneller Ebene ist aber festzustellen, dass in der Schweiz kaum eine politische Debatte über diese Alternative stattfindet und kaum akademische Forschung dazu betrieben wird. Die stärksten Strukturen sind im Waadtland und in Genf zu finden, wo sich mehrere hundert Sozialunternehmen als alternative Wirtschaftskammern organisiert haben. Ihre Kammern der sozialen und solidarischen Wirtschaft 25 dienen sowohl der Stärkung des Netzwerks als auch der Förderung eines alternativen, komplementären Wirtschaftsmodells in Politik und Gesellschaft. In der Deutschschweiz werden auch die ersten Netzwerke gegründet. ${ }^{26}$ Generell ist aber weder ein starker nationaler Verband noch eine systematische Förderung solcher Unternehmen zu finden - im Gegensatz zu den Programmen für die 'traditionelle' Wirtschaft. ${ }^{27} \mathrm{Als}$ Ausnahme dienen Projekte des Genfer Staates sowie vereinzelte Projekte auf Gemeindeebene. Zum Beispiel unterstützt die Stadt Lausanne die Gründung von Wohngenossenschaften und lokalen Geschäften im Rahmen eines neu zu bauenden Ökoquartiers. ${ }^{28}$

Ferner gibt es in der ganzen Schweiz keine Forschungseinrichtung, die sich ganz dem Sozialunternehmertum im weiteren Sinn widmet. Dies im Gegensatz zu anderen Ländern, in welchen größere Institute zu finden sind. ${ }^{29}$ Diese Lücke spiegelt sich im Ausbildungsangebot wider. Neben den

24 Vgl. Giddens, Anthony, Der dritte Weg, Frankfurt am Main, Suhrkamp, 1999.

25 Auch apres-VD und apres-GE genannt, siehe www.apres-vd.ch und www.apres-ge.ch. 2011 fand ferner das erste Treffen im Kanton Fribourg statt.

26 Zum Beispiel die Arbeitsgemeinschaft Schweizer Sozialfirmen, www.assof.ch.

27 Siehe zum Beispiel die Förderungsprogramme der Seco oder der KTI.

28 Siehe www.lausanne.ch/ecoquartier.

29 Zum Beispiel in Belgien, Italien, Frankreich, Dänemark sowie in den meisten Business Schools (Harvard, Stanford, Oxford, HEC Paris). 
unzähligen traditionellen Managementausbildungen und den wenigen Nonprofit-Management-Kursen sowie Corporate Social ResponsibilityProgrammen ist keine Sozialunternehmertum-Ausbildung zu finden. Anekdotisch kann sogar erwähnt werden, dass von 40 Studenten, die im Rahmen eines Social Entrepreneurship Seminars befragt wurde, keiner auch nur einmal etwas über die Genossenschaft als mögliche juristische Form für eine Firma hörte - trotz eines fünfjährigen Managementstudiums mit Vorlesungen über Firmenrecht, Organisation, Strategie, und Wirtschaftsethik.

2010 lehnte der Bundesrat ein Postulat über Genossenschaftsforschung im Rahmen des Internationalen Jahres der Genossenschaften (2012) ab. ${ }^{30}$ Genossenschaften sind jedoch besonders gut geeignet, um Bürgerwerte im Rahmen eines Unternehmens zu verwirklichen. ${ }^{31}$ Ein kurzer Blick in die Geschichte der schweizerischen Genossenschaften zeigt viele gelungene Kombinationen von gesellschaftlichen und wirtschaftlichen Zielen.

Sozialunternehmertum muss aber nicht auf Genossenschaften begrenzt werden. Eine Studie von Apres-GE zeigt zum Beispiel, dass nur 6\% ihrer Mitglieder Genossenschaften sind. ${ }^{32}$ Bemerkenswert ist die Möglichkeit schweizerischer Aktiengesellschaften, in ihren Statuten ethische Ziele und Werte einzubauen. Dies im Gegensatz zu anderen Ländern, die wegen strengeren gesetzlichen Profitpflichten für Firmen neue Rechtsformen schafften, um 'Community Interest Companies' (wie sie in Großbritannien heißen) zu erlauben und zu fördern.

Die Vielzahl möglicher gesetzlicher Strukturen soll nicht als Schwäche betrachtet werden. Vielmehr spiegelt sie die Idee wider, dass bei Sozialunternehmertum das Bürgerethos wichtiger ist als die Rechtsform. ${ }^{33}$ In diesem Sinn stellt sich die Frage, ob sich nicht Teile der wichtigen und

30 Das Postulat wurde im Herbst 2010 von Nationalrat Eric Nussbaumer eingereicht und fragte nach einem nationalen Bericht über Entwicklung und Rolle der Genossenschaften in der Schweiz.

31 Siehe oben.

32 Siehe Après-GE, Etude statistique. Photographie de l'économie sociale et solidaire à Genève, Genf, Chambre de l'économie sociale et solidaire Après-GE, 2010.

33 Die Rechtsform ist sogar keine Garantie dafür. Gewisse Sozialunternehmen im engeren Sinn bezweifeln zum Beispiel, dass ältere Genossenschaften wie Coop, Migros oder die Raiffeisenbank eine bürgerliche Dimension bewahrt haben. Weiterführende Studien wären allerdings notwendig, um die genauen Werten der verschiedenen Akteure zu vergleichen. 
vielfältigen sozialen Engagements vieler Familienunternehmen dem Sozialunternehmertum annähern. ${ }^{34}$ Obwohl die meisten kleinen und mittleren Unternehmen sich nicht als 'Sozialunternehmen' ansehen würden, weisen sie ein stärkeres Interesse für die lokale Gemeinschaft auf und unterstellen Profitmaximierung ihren Bürgerwerten.

Kurz gefasst können Sozialunternehmer und Sozialunternehmen im weiteren Sinn als Vorbilder einer bewussten Verflechtung des Bürgerethos mit dem wirtschaftlichen Handeln betrachtet werden. Wie in der oben genannten Gleichung ist das Unternehmen in diesem Kontext weniger eine unabhängige und wert-lose 'juristische Person'. Eher kann das Sozialunternehmen als das Ergebnis des Einsatzes von Unternehmern verstanden werden, die in ihrem Bürgerstatus die Orientierung und das Durchhaltevermögen für ihr Projekt finden.

Dadurch werden die ersten zwei Glieder der oben genannten Gleichung (das Unternehmen und der Unternehmer) neu mit dem Bürger verbunden. Es bleibt also zu untersuchen, ob das Eigentümer-Glied der Gleichung auch wieder mit dem Bürger gleichgesetzt werden kann.

\section{Die Eingliederung des Eigentümers in die Gesellschaft}

In der Schweiz können viele Beispiele eines stärkeren Bürgerethos unter Investoren gefunden werden - trotz der heutigen Skandale. Wie bei Sozialunternehmen beruhen die heutigen Initiativen auf einer längeren Tradition lokaler und solidarischer Bank- oder Versicherungsinstitutionen. Die älteren Bankgenossenschaften wie die Raiffeisenbank, aber auch jüngere und sozial aktivere Akteure wie die Alternative Bank Schweiz kennen seit den wiederkehrenden Finanzkrisen neuen Erfolg. Ferner stellt die Bank WIR mit ihrer eigenen Währung eine einzigartige alternative Finanzinstitution dar.

Dazu sollen noch die vielen nicht rein durch Profit motivierten Mikrofinanzprojekte erwähnt werden. Mikrofinanz dient dazu, vor allem Leuten aus Entwicklungsländern Finanzdienstleistungen (Sparkonto,

34 Vgl. Fassin, Yves, SMEs and the fallacy of formalising CSR, Business Ethics: A European Review, 17(4), 2008, 364-378 sowie Morsing, Mette/Perrini, Francesco, CSR in SMEs: do SMEs matter for the CSR agenda?, Business Ethics: A European Review, 18(1), 2009, 1-6. 
Darlehen aber auch Risikoversicherung) für kleinere Beträge anzubieten. ${ }^{35}$ Obwohl sich diese Investitionsfonds oft der dritten Welt widmen, sind auch Akteure auf dem Schweizer Markt zu finden. Zum Beispiel bietet die Stiftung Mikrokredit Solidarität Schwei $2^{36}$ kleinere finanzielle Darlehen für Unternehmensprojekte, die wegen ihrer begrenzten Größe oder ihres ungewöhnlichen Wirtschaftsmodells kein Interesse bei traditionellen Banken finden.

Auch unter Pensionskassen, die weiter oben als wichtige Akteure der Emanzipation von Unternehmen von der Gesellschaft erwähnt wurden, können Institutionen mit einem größeren Bürgerethos gefunden werden. Neben der berühmten Ethos-Stiftung, die sich für mehr Transparenz und Verantwortung in traditionellen Firmen einsetzt, sind auch weniger berühmte aber eher radikaler engagierte Akteure zu finden. Zum Beispiel spielt die Pensionskasse Nest ${ }^{37}$ eine wichtige Rolle in der Debatte um eine 'alternative' Wirtschaft - und weist trotz oder womöglich gerade wegen strengen sozialen und umweltfreundlichen Investitionskriterien starke finanzielle Ergebnisse auf.

\section{Schlussfolgerung}

Im ersten Teil wurde argumentiert, dass in der Wirtschaft eine dreifache Scheidung stattfand. Demzufolge kann die Bürgertugend wirtschaftliche Organisationen nicht mehr beeinflussen.

Im zweiten Teil wurden Sozialunternehmen und alternative Finanzinstitutionen als ökonomische Akteure vorgestellt, die heutzutage das Ziel erreichen, die Gleichung Unternehmen-Unternehmer-Eigentümer-Bürger in ihren wirtschaftlichen Tätigkeiten aufrechtzuerhalten.

Wie es aber von der Gleichung abzuleiten ist, kann eine solche Reintegration nur dann stattfinden, wenn die jeweiligen Personen ihre Bürgerverantwortung ernst nehmen und die Brücken zwischen den

35 Siehe zum Beispiel Eclof in Genf sowie Intercooperation in Bern. Bemerkenswert ist der Fond International de Garantie FIG, der als Garantie für Banken der Entwicklungsländer dient. Letztere Institutionen verfügen über ausreichend Kapital, zögern jedoch, es lokal zu investieren. Auch interessant ist die Zusammenarbeit zwischen Zürich Versicherung und der Internationalen Arbeitsorganisation betreffend Mikroversicherung.

36 Siehe www.mikrokredit-solidaritaet.ch

37 Siehe www.nest-info.ch. 
gesellschaftlichen Rollen neu bauen. Erste Untersuchungen in der Westschweiz zeigen, dass Individuen und ihre persönliche Weltanschauung eine entscheidende Rolle spielen, wenn ein Bürgerethos in die Wirtschaft eingebracht werden soll. Die zwei 'Kammern der sozialen und solidarischen Wirtschaft' kamen nämlich nur durch das Unternehmertum und die Beharrlichkeit einzelner Persönlichkeiten zustande. ${ }^{38}$

Es stellt sich also die Frage, ob die Lösung der heutigen Legitimitätskrise der Wirtschaft eher in der Förderung eines Bürgerethos bei Wirtschaftsakteuren $\mathrm{zu}$ finden ist als in komplexen rechtlichen Instrumenten oder allzu einfachen Social Responsibility Programmen. Sozialunternehmer als Bürger-Unternehmer fordern Akademiker, Politiker, und Wirtschaftsakteure heraus, die in der Wirtschaft herrschende Weltanschauung in Frage zu stellen. Das Modell des Homo oeconomicus, das einen egozentrischen Wirtschaftsautokraten als Grundlage des wirtschaftlichen Handelns ansieht, ist durch ein Verständnis des Menschen als einer ganzheitlichen Person zu ersetzen. Anstatt Wirtschaftsakteure als gierige und egoistische Individuen zu erziehen und eine solche Werthaltung $\mathrm{zu}$ rechtfertigen, werden akademische und politisch-administrative Institutionen aufgerufen, nach Wirtschaftsstrukturen zu suchen, die auf einem Verständnis des Wirtschaftsakteurs als Mitbürger beruhen. ${ }^{39}$ Als ganzheitliche Personen sollen Wirtschaftsakteure dazu angeregt werden, ihre Verantwortlichkeiten in allen Sphären ihres Lebens (inklusive Arbeit, Sparen und Konsum) wahrzunehmen. Dass dies im heutigen gesellschaftlichen und wirtschaftlichen Kontext möglich ist und von vielen erwartet wird, scheint der Erfolg von Sozialunternehmen zu bestätigen. In akademischen und politischen Institutionen fehlt aber noch der Wille,

38 Siehe auch Bornstein, Die Welt verändern; Maak, Thomas/Pless, Nicola M., Business leaders as citizens of the world. Advancing humanism on a global scale, Journal of Business Ethics, 88, 2009, 537-550 sowie die Kritik von Zivkovic, Sharon, Addressing Society's Most Pressing Problems by Combining the Heroic and Collective Forms of Social Entrepreneurship, presented at the $3^{\text {rd }}$ EMES Conference, Roskilde, DK, 2011.

39 Siehe Gonin, Michael, Business research, self-fulfilling prophecy, and the inherent responsibility of scholars, Journal of Academic Ethics, 5(1), 2007, 33-58 sowie Gonin, Michael/Palazzo, Guido/Hoffrage, Ulrich, Neither Bad Apple nor Bad Barrel: How the Societal Context Impacts Unethical Behavior in Organizations, Business Ethics: A European Review, forthcoming. 
diesen Wandel ernsthafter als bisher zu berücksichtigen, zu studieren, und sowohl theoretisch wie auch praktisch weiterzuentwickeln. 